Reprod. Nutr. Dévelop., 1987, 27 (1 B), 157-169.

\title{
Contrôle du comportement ingestif et mérycique par les neuropeptides
}

\author{
L. BUÉNO, P. RIVIEERE
}

Station de Pharmacologie, I.N.R.A.

180, chemin de Tournefeuille, 31300 Toulouse, France.

Summary. Neuropeptidergic control of feeding behavior and rumination in sheep.

In sheep as in other species, intracerebroventricular (ICV) administration of picomolar doses of several peptide hormones affects food intake and rumination, suggesting that these hormones play a role in the physiological control of feeding behavior.

Their effects depend on their duration (short vs long-term) and/or their nature (orexigenic vs anorexigenic). Among the short-term satiety factors, $\mathrm{CCK}_{8}, \mathrm{CRF}$ and calcitonin, administered ICV, decrease food intake by reducing the rate $\left(\mathrm{CCK}_{8}\right)$ or the duration (CRF, calcitonin) of ingestion ; in contrast, the gastrin group of peptides (gastrin 17, penta or tetragastrin) reduces food intake by promoting an early period of rumination.

Opioid peptides such as Met-enkephalin and Dynorphin initiate food intake in satieted sheep and are supposed to be active in the short-term regulation of food intake in these ruminants. In contrast both calcitonin gene-related peptide (CGRP) and growth hormonereleasing factor (GRF) increase the daily food intake, when ICV injected, but their centrally mediated orexigenic effects depend on the regimen and the digestive status.

These results confirm the species differences observed in food intake regulation and the major role of the C.N.S. in controlling feeding behavior and energy balance in ruminants.

\section{Introduction.}

L'hypothèse de l'existence d'une régulation centrale neuropeptidergique de l'appétit est récente et coïncide avec la mise en évidence pour de nombreux peptides d'un rôle de neurotransmetteurs au niveau du système nerveux central (SNC) ou périphérique. Par ailleurs, nombre de ces peptides sont localisés dans les aires du cerveau classiquement considérées comme des zones de régulation du comportement alimentaire. A l'heure actuelle la plupart de ces études, menées surtout chez le rat, sont de nature pharmacologique. Elles ne permettent qu'une approche très discutable des phénomènes de régulation physiologique. Elles peuvent néanmoins servir de base à une thérapeutique ou à des applications d'ordre zootechnique.

Chez le rat, Morley et al. en 1985 ont dénombré 15 peptides capables de diminuer la prise alimentaire après administration centrale. L'administration localisée dans l'hypothalamus a permis de les classer au même titre que ceux favorisant la prise de nourriture selon la zone d'injection. 
Chez les ruminants, la liste des peptides connus pour leurs effets sur le comportement alimentaire lorsqu'ils sont administrés par voie centrale est limitée par l'intérêt tardif porté à la connaissance de ces mécanismes régulateurs (Buéno, Hondé et Duranton, 1986).

En 1979, Della-Fera et Baile montraient que l'administration intracérébroventriculaire (ICV) de l'octapeptide terminal de la cholecystokinine $\left(\mathrm{CCK}_{8}\right)$ inhibait à court terme (quelques heures) la prise de nourriture chez le mouton. D'autres hormones du groupe de la CCK possèdent également un effet anorexigène (Hondé et Buéno, 1984) alors qu'en règle générale les peptides opioïdes (enképhalines, $\beta$ endorphine, dynorphine) augmentent la prise de nourriture (Baile et al., 1981).

Dans ce rapport nous recensons les résultats récents concernant la nature et l'origine des effets des neuropeptides, étudiés à ce jour, sur le comportement ingestif et mérycique chez les ruminants.

\section{Neuropeptides du groupe de la gastrine et de la CСК.}

Plusieurs peptides du groupe de la gastrine ont été identifiés dans des extraits d'hypothalamus de mouton, en particulier la $\mathrm{CCK}_{8}$ mais également une forme tétrapeptidique pouvant provenir de la gastrine ou de la CCK (Rehfeld, 1978). Ces formes ne semblent pas provenir de la circulation périphérique car la $\mathrm{CCK}_{8}$ marquée administrée par voie intraveineuse chez le lapin n'est pas détectée dans le liquide céphalo-rachidien (LCR) (Passaro et al., 1982). Néanmoins il a été constaté que les taux de $\mathrm{CCK}_{8}$ hypothalamiques diminuent proportionnellement à la durée du jeûne ou augmentent après le repas chez le rat (McLaughłin et al., 1985) de même que chez le mouton (Scallet, Della-Fera et Baile, 1985). En fait, il est actuellement démontré que ce peptide est synthétisé dans les neurones du cerveau et qu'il existe aussi des récepteurs spécifiques à la $\mathrm{CCK}_{8}$ du niveau du SNC et en particulier dans I'hypothalamus où elle interviendrait comme une neurohormone (Dockray et al., 1978).

Plusieurs études ont montré que la $\mathrm{CCK}_{8}$ perfusée par voie ICV à doses picomolaires chez le mouton entraînait une diminution importante des quantités ingérées pendant plusieurs'heures (Della-Fera et Baile, 1979 ; Buéno, Duranton et Ruckebusch, 1983). L'absence de modifications de la prise d'eau et de la température corporelle plaide en faveur d'un rôle spécifique. L'allongement de la durée du jeûne préalable entraîne une augmentation des doses nécessaires pour obtenir la même réduction du niveau d'ingestion suggérant que la $\mathrm{CCK}_{8}$ intervient en tant que correcteur du déficit énergique (Della-Fera et Baile, 1980). Toutefois, dans certaines conditions, les effets anorexigènes de la $\mathrm{CCK}_{8}$ sont prolongés ; tel est le cas chez le mouton adapté à ingérer sa ration quotidienne en $6 \mathrm{~h}:$ l'injection de $\mathrm{CCK}_{8}$ pendant l'une des périodes d'ingestion diminue la quantité ingérée en $48 \mathrm{~h}$ (Della-Fera et Baile, 1980). L'augmentation de la prise de nourriture dans les premières heures faisant suite à l'administration ICV d'anticorps anti-CCK $K_{8}$ apparaît comme une preuve directe du rôle physiologique de la $\mathrm{CCK}_{8}$ dans les phénomènes de satiété à court terme chez le mouton ; par ailleurs une ingestion accrue est constatée à la suite de l'administration ICV d'un bloqueur spécifique des récep- 
teurs à la $\mathrm{CCK}_{8}$ (Della-Fera et al., 1981). Le fort poids moléculaire des anticorps limite leur passage et réduit les possibilités de séquestration de la $\mathrm{CCK}_{8}$ à l'intérieur du cerveau, renforçant ainsi l'hypothèse d'une libération de $\mathrm{CCK}_{8}$ dans le liquide céphalo-rachidien (LCR). Ce peptide serait ensuite véhiculé jusqu'au niveau des sites d'action. Ainsi, lors de sa perfusion au niveau d'un ventricule latéral, des quantités non négligeables (environ $20 \%$ ) sont retrouvées au niveau de la Cisterna magna ; la $\mathrm{CCK}_{8}$ est faiblement dégradée dans le LCR et la majeure partie se fixe dans les structures paraventriculaires des $3^{e}$ et $4^{e}$ ventricules (DellaFera et al., 1981). Mais le(s) site(s) d'action et les mécanismes impliqués sont peu connus à ce jour. Enfin les peptides du groupe de la CCK agissent au niveau central pour modifier la sécrétion d'insuline (Della-Fera et al., 1981) et la motricité gastro-intestinale (Buéno, Duranton et Ruckebusch, 1983) et peuvent ainsi influencer indirectement le comportement ingestif. Toutes ces données ont amené Della-Fera et Baile (1984) à proposer un schéma indiquant les différents sites d'action de la $\mathrm{CCK}_{8}$ en fonction de son origine (fig. 1).

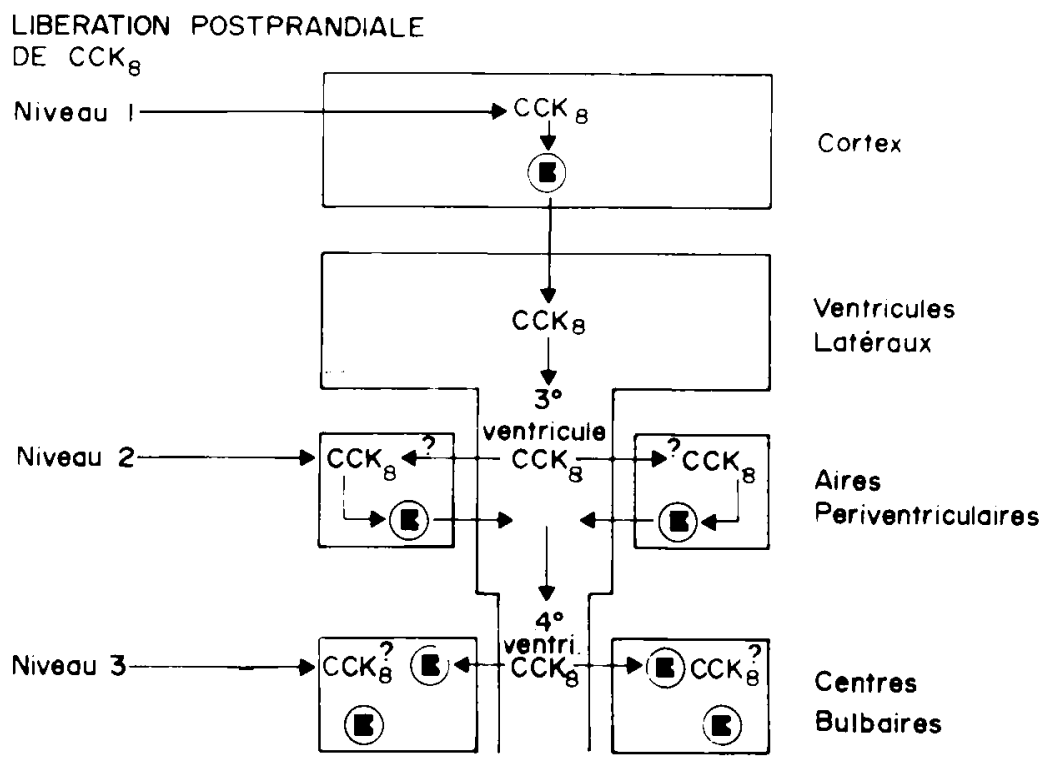

(B) Recepteurs à lo $\mathrm{CCK}_{8}$

FIG. 1. - Différents niveaux possibles d'action centrale de l'octapeptide terminal de la cholecystokinine dans la régulation de l'ingestion chez les ruminants (d'après Della-Fera et Baile, 1984).

Bien que l'administration de ces peptides conduise toujours à une réduction de l'ingestion à court terme $(3 \mathrm{~h})$, celle-ci n'est pas équivalente pour tous ces peptides (fig. 2). En effet l'action anorexigène d'une administration centrale de la $\mathrm{CCK}_{8}$ résulte chez le mouton recevant du foin d'une baisse de la vitesse d'ingestion associée à un ralentissement de la fréquence des contractions du réticulo- 
rumen (Buéno, Duranton et Ruckebusch, 1983) ; cet effet disparaît après administration préalable d'un antagoniste opiacé, la naloxone, n'ayant pas d'action propre sur le niveau d'ingestion à court terme chez le mouton. Au demeurant, la naloxone administrée à forte dose par voie intraveineuse (IV) supprime la prise de nourriture chez le mouton (Baile et al., 1981). Les effets de l'administration centrale de $\mathrm{CCK}_{8}$ et de la perfusion intra-duodénale d'acide $\mathrm{DL}$ lactique chez le mouton sont similaires : réduction de la vitesse d'ingestion, des quantités ingérées, et de la fréquence des contractions du réticulo-rumen (Buéno, 1975). L'hypothèse d'un rôle majeur de la $\mathrm{CCK}_{8}$ dans l'anorexie expérimentale consécutive à la perfusion intraduodénale d'acide $\mathrm{DL}$ lactique est vérifiée par la disparition de la baisse induite des quantités ingérées après administration centrale préalable de naloxone (Duranto et Buéno, 1986). Enfin l'activité de la $\mathrm{CCK}_{8}$ dépend de la présence du pont sylphydryl : ainsi le tétrapeptide $\mathrm{C}$-terminal de la $\mathrm{CCK}_{8}$ qui en est dépourvu, n'a plus d'effet anorexigène à court terme (Buéno, Hondé et Duranton, 1986).

- OUANTITE DE FOIN INGEREE-

$-0-30 m n-$

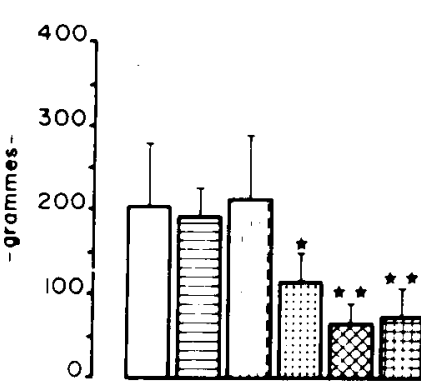

VITESSE C'INGESTION

$0.180 \mathrm{mn}-$
$-30-120 m n-$

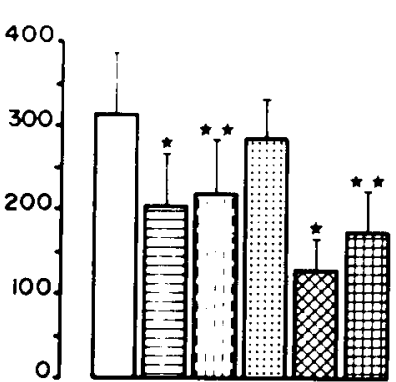

- OELAI D'APPARITION

DE LA RUMINATION

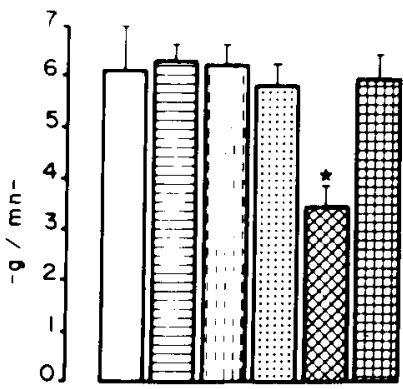

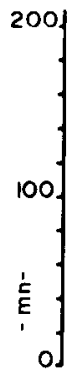

$-0-9 n-$
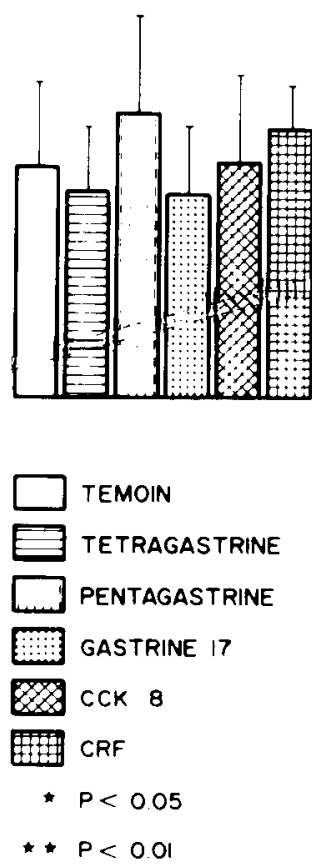

FIG. 2. - Influence de l'administration centrale (ICV), à dose isomolaires $\left(26 \mathrm{pmol}^{\mathrm{kg}} \mathrm{kg}^{-1}\right)$ de différents peptides à activité anorexigène à court terme (gastrine, $\mathrm{CCK}_{8}, \mathrm{CRF}$ ) sur les quantités ingérées, la vitesse d'ingestion et le délai d'apparition de la fre phase de rumination chez le mouton en régime foin (d'après Buéno, Hondé et Duranton, 1985).

Les autres peptides du groupe de la gastrine (gastrine 17 et pentagastrine) inhibent également la prise de nourriture lorsqu'ils sont administrés par voie ICV (fig. 2) alors qu'à doses 50 à 80 fois plus fortes ils sont inactifs par voie systémi- 
que (Hondé et Buéno, 1984). Contrairement à la $\mathrm{CCK}_{8}$, ils n'affectent pas la vitesse d'ingestion mais induisent une rumination précoce 36 à 57 min seulement après la distribution de foin au lieu de $136 \pm 28 \mathrm{~min}$; ceci explique ainsi la baisse du niveau d'ingestion de 0 à 120 min et suggère un rôle majeur de ces peptides dans l'initiation du comportement mérycique (fig. 2).

\section{Peptides opioïdes.}

Les trois familles de peptides opioïdes endogènes, enképhalines, dynorphines et endorphines ont été localisées aussi bien dans le système nerveux central qu'en périphérie. Les nombreuses études menées chez le rat n'ont pas permis cependant d'établir l'existence de variation des taux circulants liés à la prise de nourriture ou à la durée de jeûne (Baile, McLaughlin et Della-Fera, 1986). Toutefois, comme chez le rat, plusieur travaux récents suggèrent l'existence d'un contrôle central de la prise de nourriture par des peptides opiacés chez le mouton. Par exemple, l'administration ICV de méthionine-enképhaline (Met-Enk) déclenche la prise de nourriture chez le mouton rassasié alors que celle d'antagonistes (naloxone, naltrexone) injectés seuls par voie IV réduit le niveau d'ingestion à court terme (Baile et al., 1981). De plus la naloxone administrée par voie IV supprime les effets de l'administration centrale de Met-enk ou d'analogues considérés comme agonistes des récepteurs mu ou delta. La dynorphine et ses dérivés, peptides opiacés agissant spécifiquement sur les récepteurs Kappa au niveau du SNC, stimulent également la prise de nourriture chez le mouton et sont supposés être, comme chez d'autres espèces, des peptides régulateurs de l'ingestion. Ainsi la prise de nourriture peut être multipliée par 6 chez le mouton rassasié pendant les 30 minutes de perfusion ICV de 2,6 nmol. $\mathrm{min}^{-1}$ de dynorphine ${ }_{1-17}$. Néanmoins, ces peptides contiennent comme séquence $\mathrm{C}$-terminale une enképhaline la leucine-enképhaline (Leu-Enk) ; la perfusion d'un analogue de la Leu-Enk, la (D$\mathrm{Ala}^{2}, \mathrm{D}-\mathrm{Leu}^{5}$ ) enképhaline (DADL) supprime la prise de nourriture (Baile et al., 1981).

Sur la base de la spécificité de ces peptides pour une classe de récepteurs opiacés, il est possible d'attribuer chez le mouton comme chez le rat un rôle particulier à chaque type de récepteur dans le contrôle de l'ingestion (tabl. 1). Ainsi la dynorphine $_{1-17}$ principalement Kappa-agoniste et les analogues de la Met-Enk, surtout mu-agonistes, stimulent la prise de nourriture alors que la DADL, un delta agoniste, inhibe la prise de nourriture chez le mouton à jeun (Della-Fera, Buonomo et Baile, 1984). Ceci suggère l'intervention de plusieurs populations de récepteurs opiacés dans le contrôle du comportement alimentaire chez le mouton, probablement au niveau hypothalamique compte tenu des fortes concentrations en peptides opiö̈des (DiGiulio, Majane et Yang, 1979) et des récepteurs (Young et Kuhar, 1979) trouvés à ce niveau.

\section{Corticoliberine (CRF) et cortisol.}

L'augmentation de la cortisolémie qui précède la prise de nourriture spontanée et l'action stimulante sur l'appétit lors d'un traitement par les glucocorticoïdes 
(Takahashi, Inoue et Takahashi, 1977) suggèrent l'éventualité d'une régulation de la prise de nourriture par les hormones de l'axe corticotrope.

Récemment, il a été montré chez le mouton (Buéno et Duranton, 1986) que comme chez le rat (Levine et al., 1983), la corticolibérine (CRF) administrée par voie ICV (26 pmol. $\left.\mathrm{kg}^{-1}\right)$ entraîne une baisse à court terme de l'ingestion par réduction de la durée des repas. A l'opposé le cortisol administré par voie centrale à la dose de $40 \mathrm{ng} \cdot \mathrm{kg}^{-1}$ augmente de $29,4 \%$ la prise de nourriture dans les $3 \mathrm{~h}$ suivant son administration permettant même une augmentation de l'ingestion journalière pour des doses répétées.

Enfin, I'administration ICV préalable de cortisol peut inhiber les effets anorexigènes du CRF et restaurer un comportement mérycique normal (Buéno et Duranton, 1986). Les effets orexigènes du cortisol ne peuvent être dus à une réduction de la libération de CRF par "feed back " car les doses de stéroïde administrées au niveau central ne provoquent aucune variation de la cortisolémie périphérique. De plus, il a été montré que les corticoïdes agissaient au niveau hypothalamique pour favoriser la prise de nourriture induite par la noradrénaline alors que le CRF produit des effets contraires. L'ensemble de ces données suggère que le cortisol agit directement au niveau central pour modifier le comportement alimentaire et mérycique chez le ruminant indépendamment d'un rétrocontrôle sur la sécrétion d'ACTH et à un niveau différent de celui d'action du CRF.

\section{Calcitonine et CGRP (calcitonin gene related peptide).}

La calcitonine est considérée comme I'un des facteurs peptidergiques responsables de l'état de sasiété chez le rat (Freed, Perlow et Wyatt, 1979 ; Fargeas, Fioramonti et Buéno, 1984). A dose picomolaire la calcitonine inhibe la prise de nourriture chez le mouton mais, comme le CRF, sans affecter la vitesse d'ingestion ou l'apparition du comportement mérycique (Buéno, Fargeas et Julie, 1986). Par ailleurs le CGRP, peptide dérivant du même gène de synthèse que la calcitonine, effectivement produit au niveau du SNC, inhibe la prise de nourriture spontanée chez le rat (Krahn et al., 1984) et l'augmente chez le mouton (Buéno, Fargeas et Julie, 1986) lorsqu'il est administré par voie ICV (100 ng. $\mathrm{kg}^{-1}$ ). Des doses 100 fois plus élevées ne sont pas efficaces par voie systémique. Cette augmentation de l'ingestion est cependant tardive puisqu'elle est manifeste au delà de $3 \mathrm{~h}$ après administration centrale et s'accompagne d'un retard dans le délai d'apparition de la rumination. Administré par voie ICV aux doses orexigènes, juste avant l'administration centrale de calcitonine, le CGRP n'affecte pas l'activité anorexigène de cette dernière (fig. 3). Cette donnée plaide en faveur de l'existence au niveau central de récepteurs spécifiques (Fischer et al., 1985). L'action orexigène du CGRP peut également s'expliquer par une inhibition de la synthèse de calcitonine par la glande thyroïde ou par une action à long terme sur la structure des récepteurs hypothalamiques à la calcitonine. Malgré ces effets orexigènes le CGRP ralentit la fréquence des contractions du réticulo-rumen comme la plupart des peptides inhibiteurs de l'ingestion $\left(\mathrm{CCK}_{8}\right.$, gastrine, etc.). 
- OUANTITES INGEREES-

$-0.60 \mathrm{mn}-$

$-0.180 m n-$

$-0.480 \mathrm{mn}-$
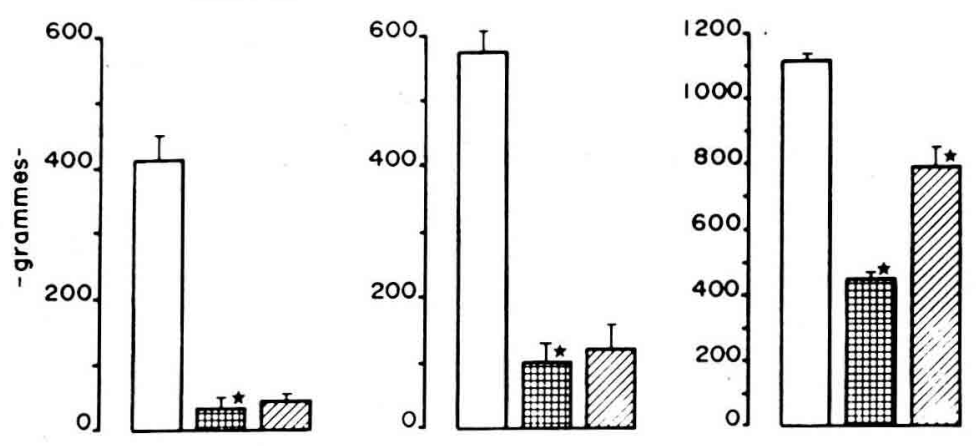

-VITESSE D'INGESTION- -DELAI D'APPARITION $-0.180 \mathrm{mn}$ DE LA RUMINATION-
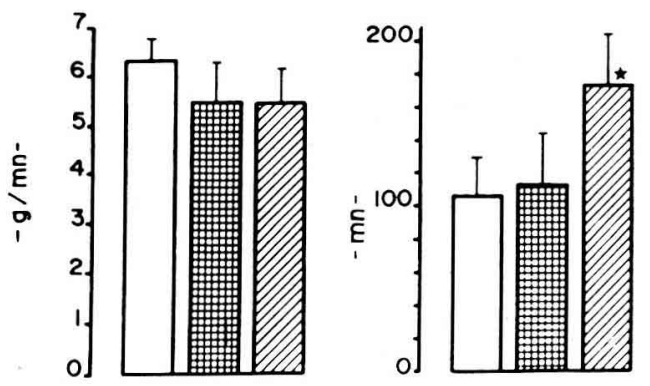

$\square$ TEMOIN

CALCITONINE(1OOMUI/kgICV)

DAS CALCITONNE(IOOMUI/kg KV) + CGRP (100ng/kg ICV)

* $\mathrm{P}<0.05$

FIG. 3. - Action antagoniste d'un traitement par le CGRP (calcitonin gene related peptides) sur les effets anorexigènes de la calcitonine administrée par voie centrale chez le mouton (d'après Buéno, Fargeas et Julie, 1986).

\section{Thyreoliberine (TRH).}

La thyréolibérine, connue pour ses effets anorexigènes chez le rat lorsqu'elle est administrée par voie centrale (Vijayan et Mc Cann, 1977), produit des effets similaires chez le mouton (Ruckebusch et Malbert, 1986). L'ingéré chute ainsi de 20 à $30 \%$ pendant les 3 premières heures du repas après administration centrale à raison de $30 \mathrm{ng} / \mathrm{kg}$. Des doses dix fois plus élevées par voie IV permettent de reproduire ces effets mais dans les deux cas les effets sont compensés dans les heures qui suivent, se traduisant par une absence de modification des quantités quotidiennes ingérées.

\section{Somatoliberine (GRF).}

A l'heure actuelle, nous ne possédons aucune donnée concernant les taux de GRF au niveau central et périphérique et encore moins les variations éventuelles 
liées au comportement alimentaire. Cependant le fait que le GRF participe au niveau central à la régulation du profil d'activité motrice postprandiale de l'intestin chez le chien (Buéno, Fioramonti et Primi, 1985) et que de fortes concentrations sont présentes dans les neurones du noyau hypothalamique ventromédian, suggèrent que ce dernier pourrait intervenir dans le contrôle général du comportement alimentaire. Ainsi récemment il a été montré que de faibles quantités 10,2 à 20 pmol) de "human pancreatic GRF " (hpGRF) et "rat hypothalamic GRF " (rhGRF) administrées par voie centrale stimulaient la prise de nourriture (Vacca-
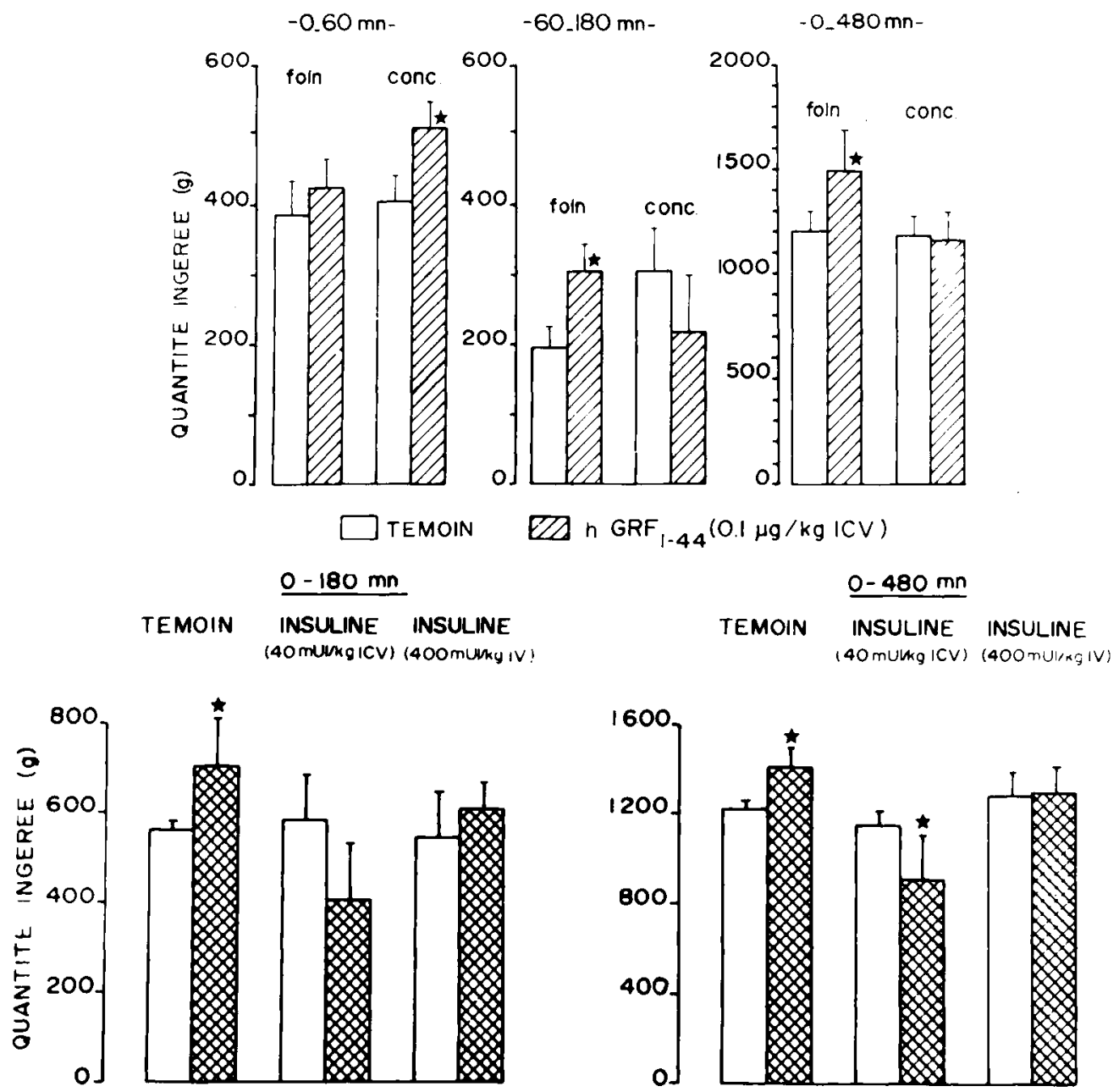

$\square$ TEMOIN

FIG. 4a, b. - Intluence du régime alimentaire et de l'insulinémie sur les effets orexigènes de l'administration centrale (ICV) de $h \mathrm{GRF}_{1-44}$ chez le mouton (d'après Rivière et Buéno, 1986). 
rino et al., 1985) alors que de plus fortes doses (1 à 4 nmol) peuvent conduire à l'anorexie chez le rat (Imaki et al., 1985). Chez le mouton des administrations isolées (tous les 3 jours), réalisées avant la distribution d'aliment le matin ont des effets différents selon le régime alimentaire. Ces effets correspondent à une augmentation passagère du niveau d'ingestion, à très court terme (1 h) pour une alimentation concentrée à base de céréales. Une administration identique pour un régime riche en fibres (foin) conduit à une augmentation plus tardive et soutenue du niveau d'ingestion due essentiellement à une élévation de la vitesse d'ingestion, se traduisant par un accroissement des quantités journalières ingérées (fig. 4a). Cette action orexigène du GRF administré par voie centrale met en jeu des neurones dopaminergiques centraux puisqu'elle est inhibée exclusivement par l'administration périphérique de substances antidopaminergiques passant la barrière hématoencéphalique telles que le métoclopramide (Buéno, Fioramonti et Primi, 1986). L'insuline apparaît de plus en plus comme une hormone intervenant au niveau central et périphérique pour contrôler la prise de nourriture chez le ruminant (Faverdin, 1985) comme chez d'autres espèces (Woods et al., 1979). Or il est connu que généralement chez le ruminant une riposte insulinique rapide ( 2 à $3 \mathrm{~h}$ après le début du repas) existe lorsqu'il reçoit un régime riche en glucides rapidement fermentescibles. Ceci pourrait expliquer l'effet orexigène limité du GRF observé avec un aliment concentré. Chez la brebis, alimentée avec du foin, l'inhibition, récemment mise en évidence, des effets du GRF après administration ICV ou IV d'insuline confirme la possibilité d'influence directe de l'insuline dans la réponse comportementale aux peptides hypothalamiques, en particulier au GRF (fig. 4b) (Rivière et Buéno, 1986).

\section{Conclusion.}

La plupart de ces données réunies dans le tableau $n^{\circ} 1$, très fragmentaires et incomplètes, soulignent cependant la possibilité d'intervention de peptides hypothalamiques ou autres neuromédiateurs du SNC dans le contrôle central du comportement ingestif et mérycique chez le mouton.

L'hypothèse d'une non spécificité des effets pharmacologiques observés, telle une aversion non spécifique pour l'aliment, ne peut être retenue en raison de la diversité des modes d'action observés. Les peptides à activité réductrice de l'ingestion peuvent en effet agir en réduisant la durée d'ingestion (CRF, calcitonine) ou la vitesse d'ingestion $\left(\mathrm{CCK}_{8}\right)$ ou encore en initiant une rumination précoce (gastrine et $\mathrm{CCK}_{4}$ ). L'augmentation de l'ingestion peut également résulter d'une durée (peptides opioïdes) ou d'une vitesse (GRF) d'ingestion accrues ou encore d'une rumination retardée (CGRP). Ces peptides peuvent également être classés selon des critères d'apparition de leurs effets (tabl. 2).

Malgré le nombre croissant de peptides trouvés actifs sur le comportement alimentaire des ruminants comme chez d'autres espèces, il apparaît important de répondre à l'heure actuelle aux questions découlant des travaux présentés : préciser les relations existant entre ces neuropeptides et les neuromédiateurs classiques (catécholamines), déterminer leur rôle dans la régulation de la balance éner- 


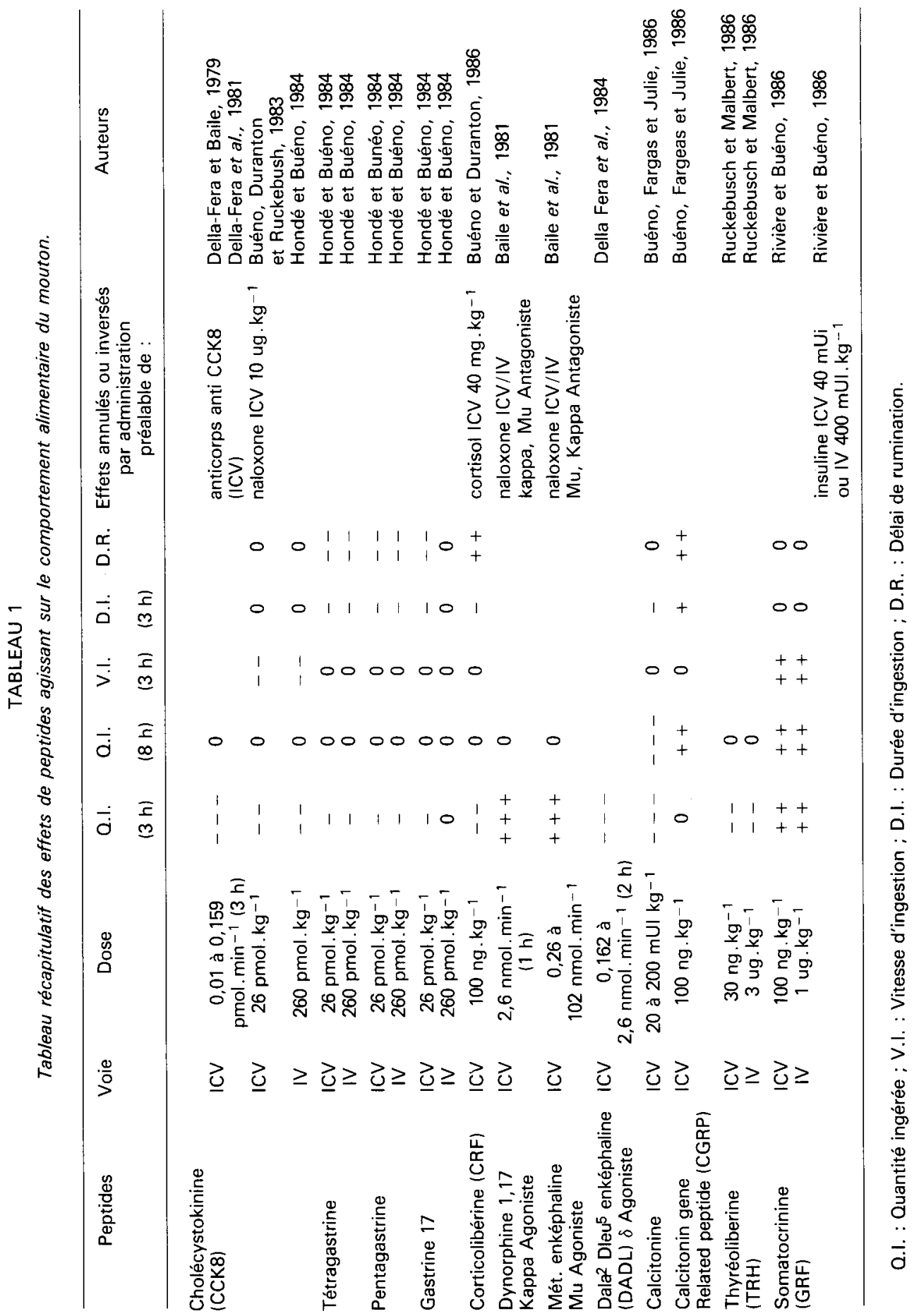


TABLEAU 2

Classification des peptides actifs par voie centrale sur le niveau d'ingestion chez le mouton selon la nature et le délai d'apparition de leurs effets.

Peptides actifs à court terme $(<3 \mathrm{~h})$

(indépendamment du régime et/ou de l'état digestif)

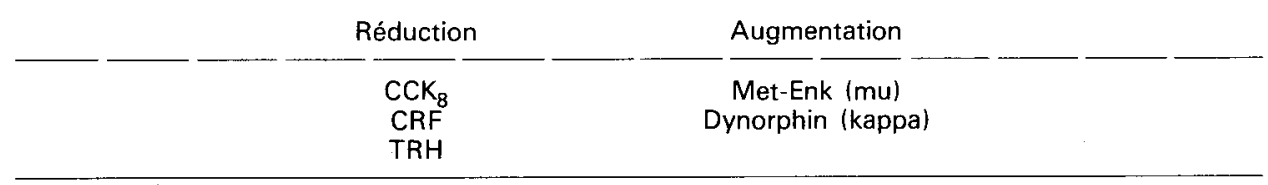

Peptides actifs à long terme $(>3 \mathrm{~h})$

(fonction du régime et/ou de l'état digestif)

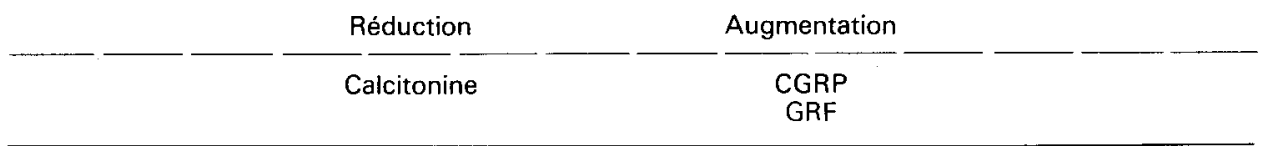

gétique et leur participation dans les phénomènes d'anorexies d'étiologie variée (parasitisme gastrointestinal, déséquilibres alimentaires, etc.). Enfin il convient également de préciser les interactions entre les divers systèmes peptidiques ; en effet, l'action anorexigène de la $\mathrm{CCK}_{8}$ est bloquée par la naloxone, un antagoniste opiacé, et la production de $\beta$-endorphines hypophysaires dépend de la cortisolémie, elle-même fonction de la sécrétion de CRF.

\author{
$2^{e m e s}$ Journées sur la Nutrition et l'Alimentation \\ des Herbivores, I.N.R.A., Paris, 19 et 20 mars 1986.
}

\title{
Références
}

BAILE C. A., KEIM D. A., DELLA-FeRA M. A., McLAughliN C. L., 1981. Opiate antagonists and agonists and feeding in sheep. Physiol. Behav., 26, 1019-1023.

BAILE C. A., McLAUGHLIN C. L., DELLA-FERA M. A., 1986. Role of cholecystokinin and opioid peptides in control of food intake. Physiol. Rev., 66, 172-223.

BUÉNO L., 1975. Rôle de l'acide DL-lactique dans le contrôle de l'ingestion alimentaire chez le mouton. Ann. Rech. vét., 6, 325-336.

BUÉNO L., DURANTON A., 1986. Effects of central administration of cortisol and corticotropin releasing factor on feeding behavior in sheep. Am. J. vet. Res. (sous presse).

BUÉNO L., DURANTON A., RUCKEBUSCH Y., 1983. Antagonistic effects of naloxone on CCKoctapeptide induced satiety and rumino-reticular hypomotility in sheep. Life Sci., 32. 855-863.

BUÉNO L., FARGEAS M. J., JULIE P., 1986. Comparative effects of calcitonine and CGRP of food intake and forestomach (reticulum) motility in sheep. Physiol. Behav., 36, 907-911.

BUÉNO L., FIORAMONTI J., PRIMI M. P., 1985. Central effects of growth hormone-releasing factor (GRF) on intestinal motility in dogs: involvement of dopaminergic receptors. Peptides, 6, 403-407.

BUENO L., HONDÉ C., DURANTON A., 1986. Influence centrale de quelques neuropeptides sur l'ingestion et l'apparition de la rumination chez le mouton. Rep. Nutr. Dév., 26, 379-380.

DELLA-FERA M. A., BAILE C. A., 1979. Cholecystokinin octapeptide continuous picomolar injections into the cerebral ventricles suppress feeding. Science, 206, 471-473. 
DELLA-FERA M. A., BAILE C. A., 1980. CCK-octapeptide injected in CSF decreases meal size and daily food intake in sheep. Peptides, 1, 51.

DELLA-FERA M. A., BAILE C. A., 1984. Control of food intake in sheep. J. anim. Sci., 59, 1362-1368.

DELLA-FERA M. A., BAILE C. A., SCHNEIDER B. S., GRINKER J., 1981. Cholecystokinin antibody injected in cerebral ventricles stimulates feeding in sheep. Science, 212, 687-689.

DELLA-FERA M. A., BUONOMO F. C., BAILE C. A., 1984. Opioid control of growth hormone secretion and food intake in sheep. Fed Proc., 43, 630.

DigIULIO A. M., MAJANE E. M., YANG H. Y. T., 1979. On the distribution of Met5 and Leu5 enkephalins in the brain of the rat, guinea pig and calf. Brit. J. Pharmacol., 66. 297.

DOCKRAY G. J., GERGORY R. A., HUTCHISON J. B., 1978. Isolation, structure and biological activity of two cholecystokinin octapeptides from sheep brain. Nature (London), 274, 711.

DURANTON A., BUÉNO L., 1986. A passible central opiate mecanism involved in the inhibition of food intake and reticular motility by duodenal DL-lactic acid infusion in sheep. Am. J. vet. Res., 44, 802-805.

FARGEAS M. J., FIORAMONTI J., BUÉNO L., 1984. Prostaglandins E2. A neuromediator in the central control o gastrointestinal motility and feeding behavior by calcitonin. Science, 225 , 1050-1052.

FAVERDIN P., 1985. Regulation de l'ingestion des vaches laitières en début de lactation : Etude du rôle de l'insuline. Thèse Doct. Ing. Univ. Paris-Grignon, $131 \mathrm{p}$.

FISCHER J. A., HENKE H., PETERMANN J., ISCHOPP F. C., 1985. Calcitonin gene related peptide (CGRP) and calcitonin (CT) and their binding sites in the central nervous system 185-192. In A. PECILE, Int. Symp. Calcitonin, Elsevier Sci. Publ. BV.

FREED W. J., PERLOW M. J., WYATT R. J., 1979. Calcitonin inhibitory effect on eating in rats. Science, 206, 850-852.

HONDÉ C., BUÉNO L., 1984. Evidence for central neuropeptidergic control of rumination in sheep. Peptides, 5, 81-83.

IMAKI T., SHITASAKI T., HOTTA M., MASUdA A., DEMURA H., SHIZUME K., LING N., 1985. The satiety effect of growth hormone-releasing factor in rats. Brain Res., $\mathbf{3 4 0}$, 186-188.

KRAHN D. D., GOSNELL A., LEVINE A. S., MORLEY J. E., 1984. Effects of calcitonin generelated peptide on food intake. Peptides, 5, 861-864.

LEVINE A. S., ROGERS B., KNEIP J., GRACE M., MORLEY J. E., 1983. Effect of centrally administered corticotropin releasing factor (CRF) on multiple feeding paradigms. Neuropharmacol., 22, 337-339.

McLAUGHLIN C. L., BAILE C. A.; DELLA-FERA M. A., KASSER T. G., 1985. Mealstimulated increased concentration of CCK in the hypothalamic of Zucheiokese and lean rats. Physiol. Behav., 35, 215-220.

MORLEY J. E., LEVINE A. S., GOSNELL B. A., KRAHN D. D., 1985. Peptides as central regulators of feeding. Brain Res. Bull., 14, 511-519.

PASSARO E., DEBA S. H., OLDENDORF W., YAMADA T., 1982. Rapid appearance of intraventricularly administered neuropeptides in the peripheral circulation. Brain Res., 241, 335-340.

REHFELD J. F., 1978. Immunochemical studies on cholecystokinin II. Distribution and molecular heterogenecity in the central nervous system of man and hog. J. biol. Chem., 253, 4022-4030.

RIVIĖRE P., BUÉNO L., 1986. Influence of regimen and insulinemia on orexigenic effects of GRF $_{1-44}$ in sheep. Physiol. Behav. (submitted).

RUCKEBUSCH Y., MALBERT C. H., 1986. Stimulation and inhibition of food intake in sheep by centrally-administrated hypothalamic releasing factors. Life Sci., 38, 929-934.

SCALLET A. C., DELLA-FERA M. A., BAILE C. A., 1985. Satiety hunger and regional brain content of cholecystokinin gastrin and Met-Enkephalin immunoreactivity in sheep. Peptides, 6, 937-943.

TAKAHASHI K., INOUE K., TAKAHASHI Y., 1977. Parallel shift in circadian rhythms of adrenocortical activity and food intake in blinded and intact rats exposed to continuous illumination. Endocrinology, 100, 1097-1107. 
VACCARINO F. J., BLOOM F. E., RIVIER J., WALE W., KOOB G. F., 1985. Stimulation of food intake in rats by centrally administered hypothalamic growth hormone-releasing factor. Nature, 314, 167-168.

VIJAYAN E., McCANN S. M., 1977. Suppression of feeding and drinking activity in rats following intraventricular injection of tyrotropin releasing hormone (TRH). Endocrinology, 100. 1727-1730.

WOODS S. C., LOTTER E. C., MCKY L. D., PORTE D., 1979. Chronic intracerebroventricular infusion of insulin reduce food intake and body weight of baboons. Nature, 282, 503-505.

YOUNG W. S., KUHAR M. J., 1979. A new method for receptor autoradiography. ${ }^{3} \mathrm{H}$ opioid receptors in rat brain. Brain Res., 179, 255. 